\title{
Satellite Attitude Tracking for Earth Pushbroom Imaginary with Forward Motion Compensation
}

\author{
Abbas Abdollahi ${ }^{1}$, Mohamad Reza Dastranj ${ }^{2}$ and Amin Reza Riahi ${ }^{3}$ \\ ${ }^{1}$ Department of Electrical Engineerin East Tehran Branch, \\ Islamic Azad University, Tehran, Iran \\ ${ }^{2}$ Department of Electrical Engineerig sabzevar Branch, \\ Islamic Azad University, sabzevar, Iran \\ ${ }^{3}$ Department of Electrical Engineerig Gonabad Branch, \\ Islamic Azad University, Gonabad, Iran
}

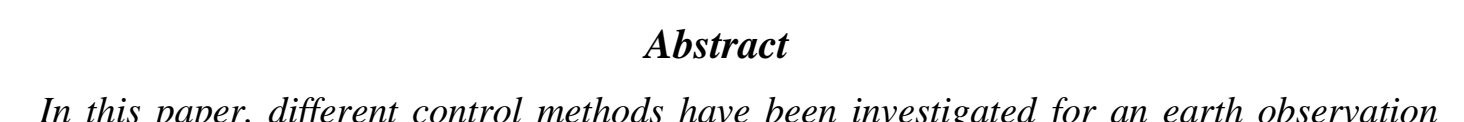

In this paper, different control methods have been investigated for an eartb observation satellite with push broom imaging. In pushbroom Imaging scan method, the satellite should be able to follow a certain path. Forward Motion Control methodhas been used to improve image quality. Due to the need for fine pointing and muld attitude maneuvers, reaction wheels are chosen as the control actuator. In this paper, PID, Fuzzy and extended LQR controllers is designed for a nonlinear model of a satellite. Research results show that controller with PID Quaternion feedback has average precision with lesscomputation. LQR controller with the extension of the cost function has the best tracking accuracy. Fuzzy controller generates Minimum control torque in comparison thers. •

Keywords: Attitude control, Pushbroom, Forward Motion Control, LQR, and Fuzzy

\section{Introduction}

New space missions for development of laser communications and high-resolution earth observation satellites require high precision pointing in their missions. Fine pointing refers to the direction of the satellite, the camera sensor or antenna toward the target location or the specified inertial. Earth pointing refers to the orientation to the ground targets, and Inertial pointing is the orrentation to other space target such as stars [1]. The military spacecraft mission for rapidly acquiring and accurately tracking a terrestrial or space-based target is often referned to as acquisitions, tracking and pointing. Civilian spacecraft such as earth observation satellite are not typically designed for such missions, but military spacecraft often must perform these missions with shared design heritage. Even considering a rigid spacecraft, rapid maneuvers with quick settling time and fine target tracking are quite challenging. Systematically consider rapid slew maneuvers as one challenge and target tracking as a second challenge.

Earth observing satellites have been developed and successfully operated for scientific, commercial and military purposes since the $1970^{\text {"c }}$ [5]. "How changes earth?" is one of most important strategic issues that have led to the development of the earth observing satellite 
research. These satellites usually use large scale digital cameras Technology based on semiconductor materials. Earth imaging missions with high precision imposes exact requirements on the attitude control system based on pointing accuracy. These requirements dictates a robust and high performance design for Rapid detection of target and high rates of image scan lines.

Satellite limitations implied that algorithm and control system be as simple and fast with low hardware and software cost. This problem is considered as a challenge for design engineers. Mission requirement for fine pointing affect the Control method, class, type and number of sensors and actuators in the attitude control and determination system ADCS. Reaction wheels for smooth and continuous control with the least amount of disturbance torque is preferred in attitude control system with fine pointing and medium-speed maneuvers requirement.

Recent work on attitude control Include linear and nonlinear control methods, fuzzy controllers, neural network method, LQR and adaptive controllers. Tisa has investigated various control methods for FalconSat-3 satellite with magnetic aetuator/and gravity gradient boom [7]. Many fuzzy logic controller applications for satelite attitude control have been proposed successfully. Steyn used three multiple input single output (MISO) fuzzy controllers for attitude stabilization of a small satellite in a Now Earth orbit, proving that the fuzzy controller solves the control constraint problenpy choosing the best magneto-torque, polarity, and switching instances [8].

In this paper fine pointing requirements has been introduced for an imaging satellite with pushbroom cameras, and mission scenario for attitude control system is proposed. Reference attitude path for imaging with FMC method is generated and attitude tracking around this reference path has been done. The evaluation of $\mathrm{Q} Q \mathrm{R}$, fuzzy and quaternion feedback control controllers is performed.

\section{Pushbroom Imaging}

In the past decades, aerial mapping were based on large cameras. Old systems had low resolution power and long Access times. At the end of the last Century a transition from analytical to digital photogrammetry was taken, then Digital cameras in large scale and based on CCD (charge-coupled device) were introduced. And using of these cameras is strongly growing.

Today earth observing satellites mainly carry pushbroom cameras. In general terms pushbrom cameras consist of an optical systems projecting an image onto a linear array of sensors. at any time only those points are imaged that lie in the plane defined by the optical center and the line containing the sensor array. This plane will be called the instantaneous plane or simply view plane [8]. 


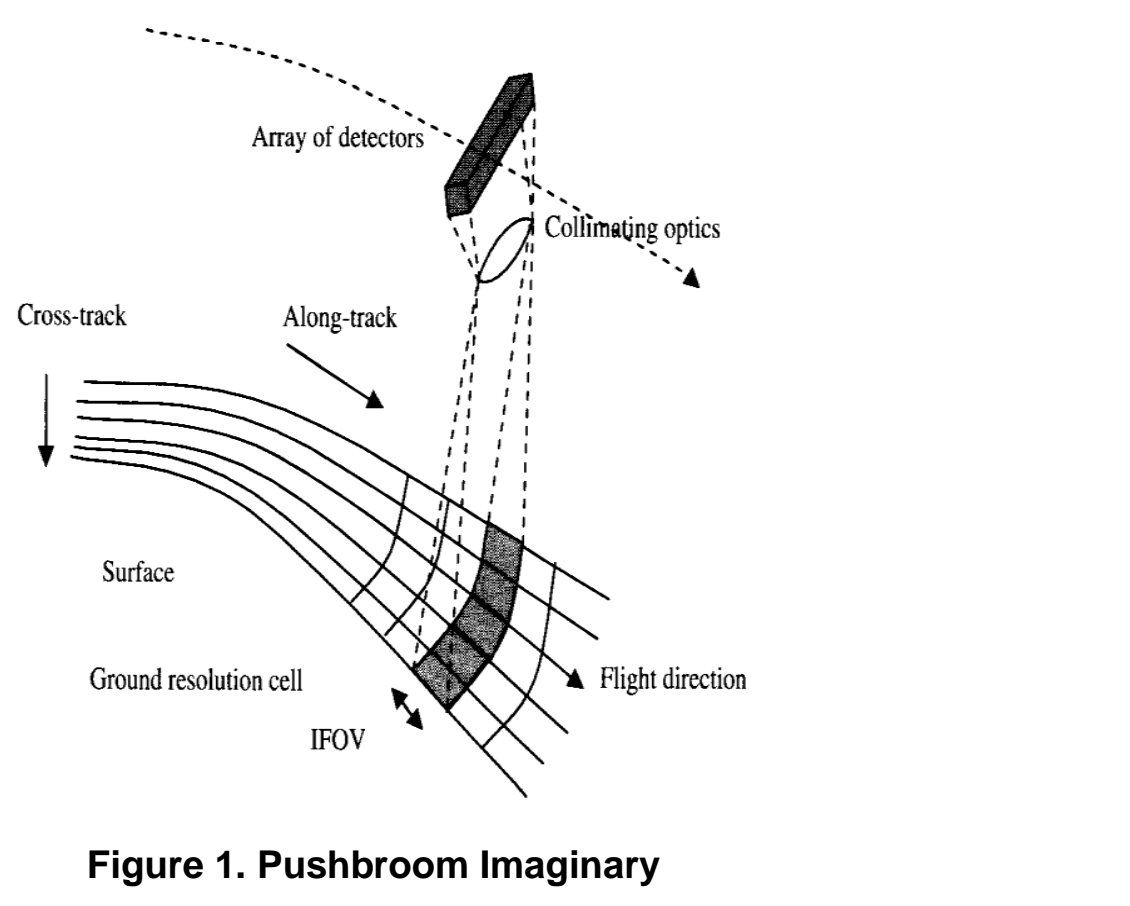

Figure 1. Pushbroom Imaginary

During such imaging pass, the satellite's attitude has to be controlled so that the bore-sight sweeps out equal distances for each image, row. The process of controlling the satellite for differing integration times, is called Forward Motion Compensation (FMC) [18]. In this imaging procedure with FMC, satellite uses larger orbital period to complete the scanning of the same area.

For attitude control system with fine pointing accurate and medium-speed maneuvers, reaction wheels for smooth and continuous control with the least amount of disturbance torque is preferred [3].

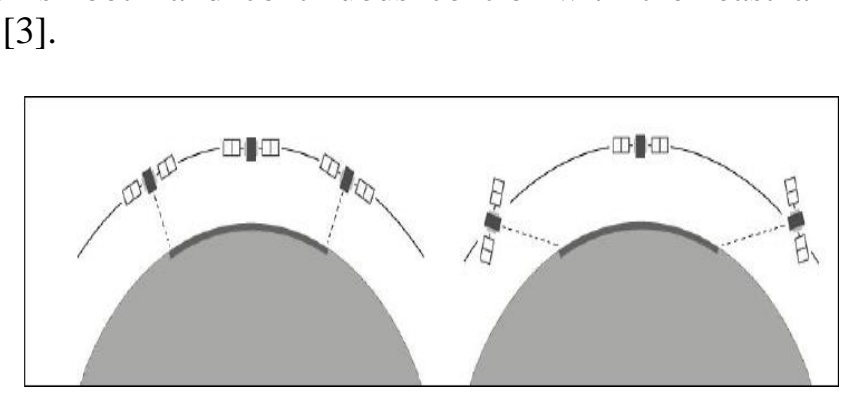

\section{Figure 2 Satellite Imaging passes. (lef) Nadir Pointing (right) FMC}

\section{Modelling}

Spacecraft is assumed to behave as a point mass model for orbital dynamics. According to Newton-Euler formulation angular momentum changes according to applied torque. With these assumptions, Satellite dynamic model can be expressed by Equation 1:

$$
I \dot{\omega}_{b i}^{b}+\omega_{b i}^{b} \times\left(I \omega_{b i}^{b}\right)=\tau^{b}
$$

Where I is is the moment of inertia, $\omega_{b i}^{b}$ is the angular velocity of the body frame with respect to the inertial frame in the body frame, $\tau^{b}$ is the torques acting on the satellite in body 
frame, and $\tau^{b}=\tau_{\text {grav }}^{b}+\tau_{w w}^{b} . \tau_{\text {grav }}^{b}$ is the gravitational torque on the satellite body, and $\tau_{w w}^{b}$ is the reaction wheel torque.

Kinematics of the satellite describes the orientation of the satellite. Kinematics is simply integration of the angular velocity. Based on quaternion parameterization of attitude kinematics, the kinematic model of a Spacecraft is according to equation (2).

$$
\frac{d}{d t} q=\frac{1}{2}\left[\begin{array}{cccc}
0 & \omega_{3} & -\omega_{2} & \omega_{1} \\
-\omega_{3} & 0 & \omega_{1} & \omega_{2} \\
\omega_{2} & -\omega_{1} & 0 & \omega_{3} \\
-\omega_{1} & -\omega_{2} & -\omega_{3} & 0
\end{array}\right] q
$$

Where $q \in \square^{4}$ is the unit norm vector of Quaternion parameters and selected to eliminate singularities present in the Euler angles. [ $\omega 1 \omega 2 \omega 3$ ] is the angular felocity vector of the body frame relative to the orbit frame and can be obtained from (3):

$$
\begin{aligned}
& \boldsymbol{\omega}_{B O}^{B}=\left[\begin{array}{lll}
\omega_{1} & \omega_{2} & \omega_{3}
\end{array}\right]=\boldsymbol{\omega}_{B I}^{B}-R_{O}^{B} \boldsymbol{\omega}_{o l}^{o} \\
& \boldsymbol{\omega}_{o l}^{o}=\left[\begin{array}{lll}
0 & -\omega_{o} & 0
\end{array}\right], \omega_{o}=\sqrt{\left(\hat{R}_{o}\right)}
\end{aligned}
$$

Where, $[0-\omega o 0]$ is the angularvelpcity of the orbity frame relative to the ECI frame, and $R_{o}^{B}$ rotation matrix between Orbit and Body frame? $\mu=3.986 \times 1014 \mathrm{~m} 3 / \mathrm{s} 2$ is the Earth's gravitational coefficient, $\mathrm{R} 0$ is the distance from Earth's center $(\mathrm{m})$. To return to Euler angles from quaternion attitude, equation 4 to 6 can be used:

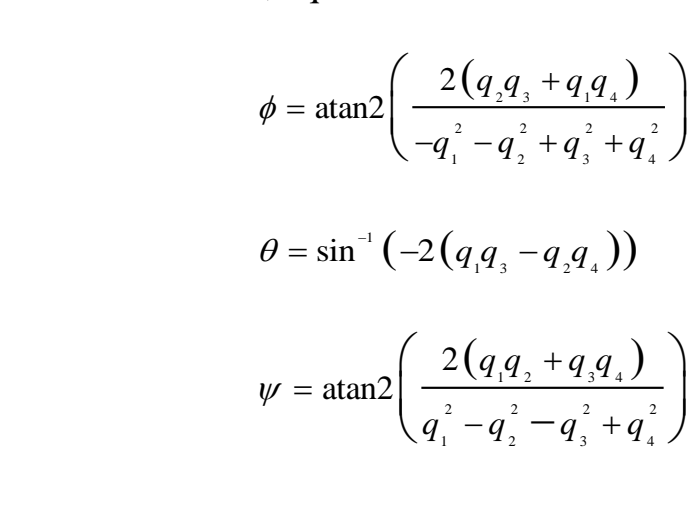

Where atan 2 is a tangent function with bounds between $-180^{\circ}$ and $180^{\circ}$ instead of $-90^{\circ}$ and $90^{\circ}$

\section{Actuator}

An actuator is the mechanism that supplies control torque for the attitude control system. Two key factors in selecting the attitude control actuator is a) pointing accuracy and b) slew maneuvers Rate requirement [6]. military operational requirements for slew from one target 
to another several times demand large angle, rapid slew maneuvers as quickly as possible and also require quick settling with minimal target tracking errors While The civilian spacecraft such as Hubble Space Telescope needs to point extremely accurately with medium slew maneuvers [2]. For accurate attitude control systems and moderately fast maneuvers, the reaction wheels are preferred because they allow continuous and smooth control with the lowest possible parasitic disturbing torques. Reaction wheels are simple disks (rotors) that are spun by an electric motor. When the motor applies a torque to speed up or slow down the rotor, it produces a reacting torque on the body of the satellite the level of torque that can be achieved with reaction wheels is of the order of 0.05-2 N-m. With control moment gyros (used in manned spacecraft), torques of $200 \mathrm{~N}$-m are achievable. However, such CMGs are very heavy and are seldom used in the ACS of ordinary-sized satellites.

\section{The Reference Path}

Research earth observation satellites for imaging with new generation of difital cameras based on CCD technology, have special requirements on earth pointing missions. During the imaging process, attitude euler angles should be managed so that, Cameras moynted on the satellite Has always been to the desired location, and earth is viewed in longer time. For fulfillment of such imaging mission, satellite must be able to execute Stabilization, maneuverability and tracking missions with high accuracy.

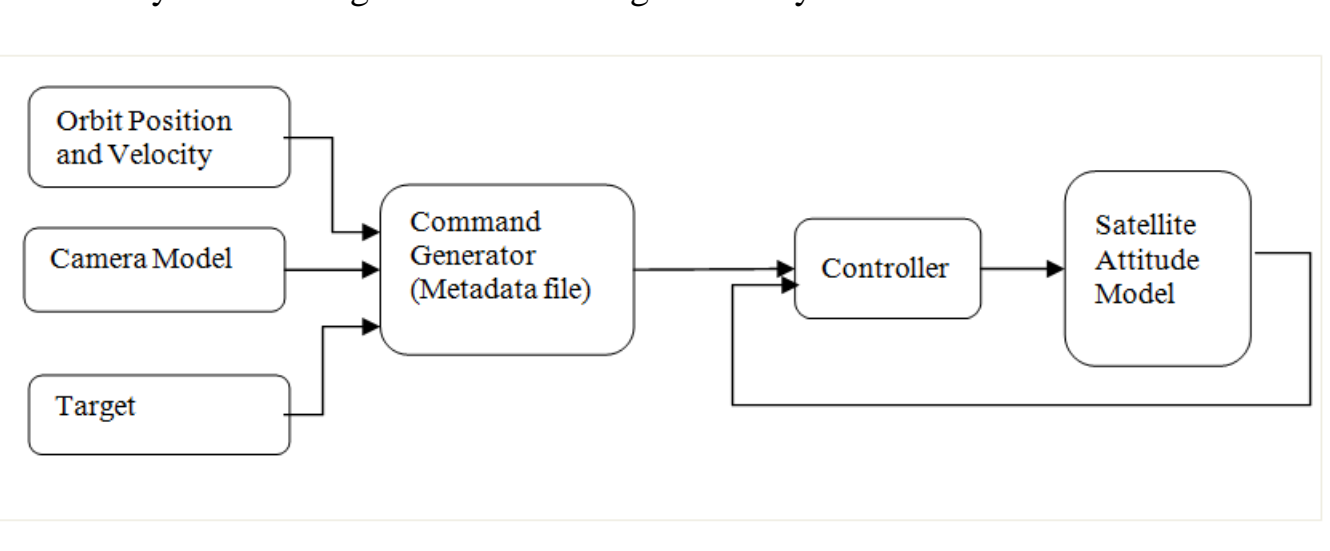

Figure 3. Satellite tracking system

\section{Controller Design}

According to Wertz definition, Attitude control is the process of achieving and maintaining an orientation inspace. An attitude maneuver is the process of reorienting the spacecraft from one attitude to another. Attitude stabilization is the process of maintaining an existing attitude relative to some external relative frame. The paper reviews various control techniques (classical control, modern and Fuzzy) for satellite attitude control with reaction wheels.

\subsection{Quaternion Feedback Control}

Quaternion Feedback Control is a classic Proportional-Integral-Derivative Feedback Control based on Quaternion error. PID Controllers have always been of interest to control engineers because of the simplicity of designing, building and maintenance, effective performance in tracking and disturbance rejection and not based on an accurate model of the system. PID Controllers form the majority of industrial controllers. Hence efforts have been made to apply these controllers to multivariable systems. If a SISO PID controller is designed 
for each input- output channel the resulting controller is a diagonal PID controller. Although can achieve some of the desired performance features with this controller, However, it should be noted that due to the diagonal nature of this controller, high performance in the face of complex dynamic systems and multivariate interference will not show up. Considering the complex dynamics of multivariable systems, it is necessary that the controller coefficient matrices P, I, D, must be non-diagonal. Quaternion error is obtained according to equation (7).

$$
q_{e}=\left[\begin{array}{cccc}
q_{4 c} & q_{3 c} & -q_{2 c} & -q_{1 c} \\
-q_{3 c} & q_{4 c} & q_{1 c} & -q_{2 c} \\
q_{2 c} & -q_{1 c} & q_{4 c} & -q_{3 c} \\
q_{1 c} & q_{2 c} & q_{3 c} & q_{4 c}
\end{array}\right]\left[\begin{array}{l}
q_{1} \\
q_{2} \\
q_{3} \\
q_{4}
\end{array}\right]
$$

In this method, the control law is calculated with equation (8).

$$
T_{c}=-K_{p} q_{e 1-3}-K_{i} \int q_{e 1-3}-K_{d} \dot{q}_{e 1-3}
$$

\subsection{LQR Controller}

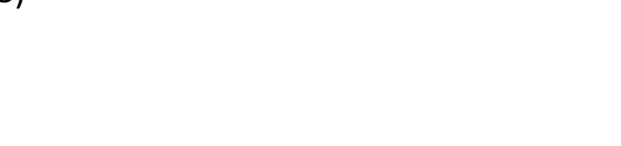

In analysis and design of control systems, state space method is considered newer than classical transfer function. In this method, the optimal control is ja major effort. The main feature of this method is that it can model systems such as linear and nonlinear systems, timevarying (or constant time), univariate and multivariable system, in the same way. On the other hand, appropriate state space representation for the idea of using Lyapunov stability and closed-loop stability using the Lyapunoy theory controller design in state space to be stronger.

Optimization methods for stabilizing the systêr ground equilibrium point, is linear time varying law based on states of System. Linear Quadratic Regulator (LQR) is a control technique used in attitude stabilization. The main idea of the control system is to find a cost function and minimize this cost function. First of all, system dynamics is linearized around the .Selected points the cost banction is minimized, and lastly, system states are fed back by a gainmatrix. A linearized form of the equations is needed for controller design. Linearized state space model of a dynamical system expressed is with equation (9).

$$
\dot{x}(t)=A(t)+B(t) u(t)
$$

Attitude system state vector is:

$$
Q(t)=\left[\begin{array}{llllll}
q_{1} & \dot{q}_{1} & q_{2} & \dot{q}_{2} & q_{3} & \dot{q}_{3}
\end{array}\right]
$$

Reference input In regulator or stabilizer systems is considered zero and the stabilization of the closed-loop is conducted by pole placement. Pole placement is a state-space design technique used to assign closed-loop poles in Multiple-Input Multiple-Output (MIMO) systems. The optimal state feedback gain matrix (compromise between states speed and control inputs) is extremely important. Cost function in the regulator problem for control input and state variables is defined in equation (11).

$$
J=\frac{1}{2} \int_{t_{0}}^{T}\left[\bar{x}^{T} \mathbf{Q} \bar{x}+\bar{u}^{T} \mathbf{R} u\right] d t
$$


$\mathrm{Q}$ is the positive semi definite weight matrix for the state deviation and $\mathrm{R}$ is the positive definite weight matrix for the actuator. In order to solve this though cost function, Ricatti equation is applied. Below Ricatti equation is given:

$$
-K A-A^{T} K-Q+K B R^{-1} B^{T} K=0
$$

The control input law is obtained from equation (13):

$$
u(t)=-K x(t)
$$

In tracking systems in addition to stabilizing, the control system must follow a reference $\bullet$ input as well. In this systems reference input is not zero and control system must be designed so that the output of the closed loop system follow the reference input. For example, the automatic pilot system in guided missiles that must follow the guidance commands. There are several methods for tracking based on state space. Since the open-loôp system is inherently integrator and has poles on imaginary axis, approach can be considered as a tracker is extended of linear regulator problem by selecting a new cost function that also include reference state. The new cost function is presented in equation (14).

$$
J=\frac{1}{2} \int_{t_{0}}^{T}\left[(x-\tilde{x})^{T} Q(x-\tilde{x})+u^{T} R u\right]
$$

And $\tilde{x}$ is the reference trajectory (desired . With the selection extended cost function for the linear regulator and to zero difference between state variable and reference values, the regulator is converted to the tracker.

\subsection{Fuzzy controller}

Fuzzy logic was first proposed by Lotfi A. Zadeh of the University of California at Berkeley in a 1965 paper.

Designed Fuzzy controller consists of three two-input one-output MISO PD fuzzy controller to drive MIMO satellie attitude system. The controller inputs are error and error rates of three angles Roll, Pitch and Yaw angles relative to the reference input. Output of these three controllers is three torques to achieve the desired attitude. For simplicity of calculation, triangular-shaped membership functions is used for fuzzy input variables figure (4) shows membership function for angle error and figure (5) shows membership function for angle error rates. Output membership functions have shown in figure (6) and consist of 7 fuzzy sets (from Very Big to Very Small). The maximum range in output member function is determinate by actuator ability in produced torque.

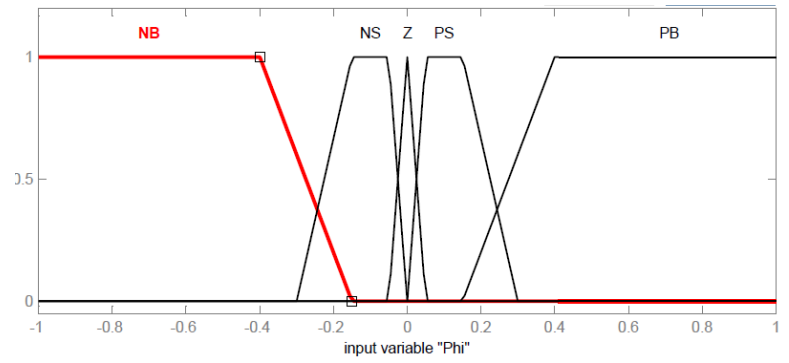

Figure 4. Member function of euler angle error 


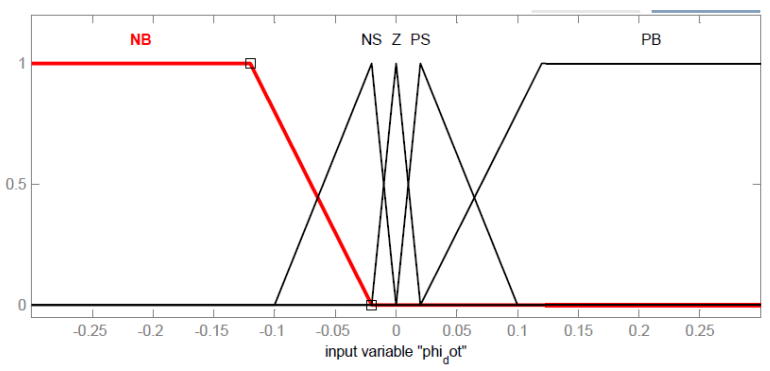

Figure 5. Member function of euler angle error rate

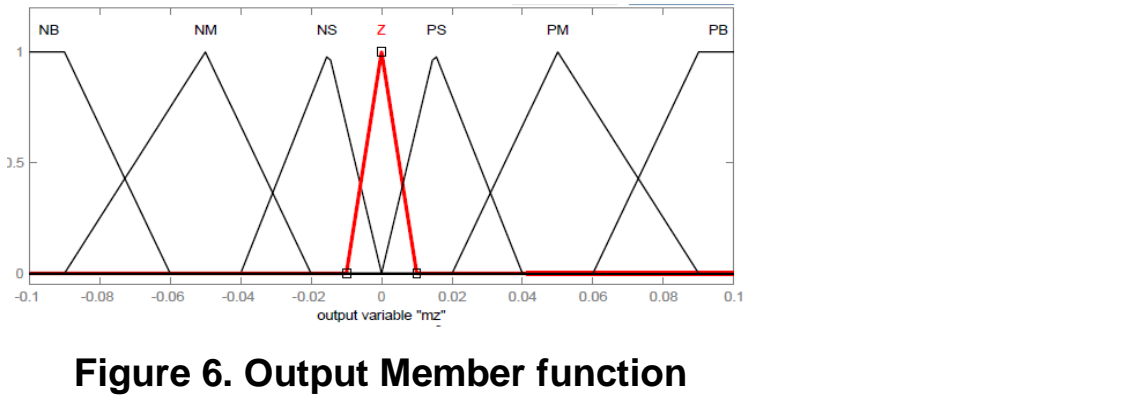

\section{Simulation}

Nonlinear satellite model with control actuator, reaction wheel is implemented in MATLAB software. Some simulation parameters are given in Table 1. Simulation results are shown in Figures 7 to 9.

\section{Table 1. Simulation Parameter}

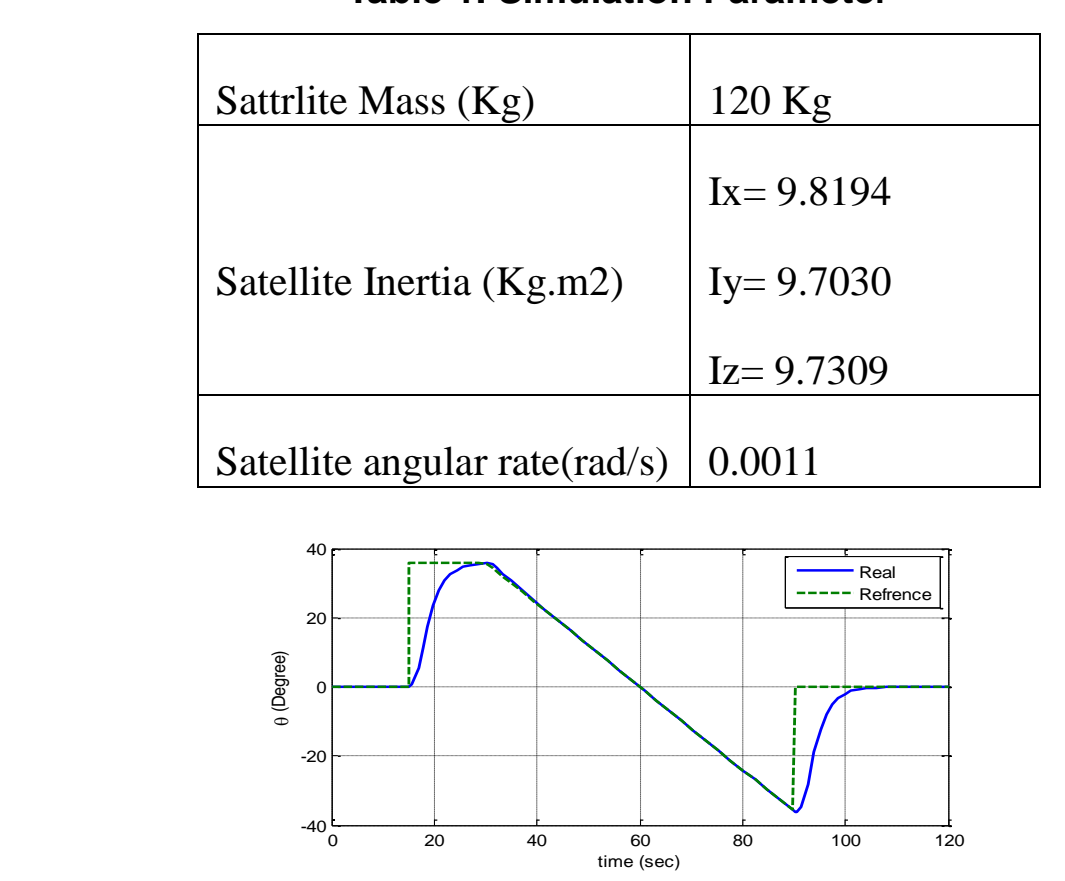

Figure 7. Quaternion feedback controller response 


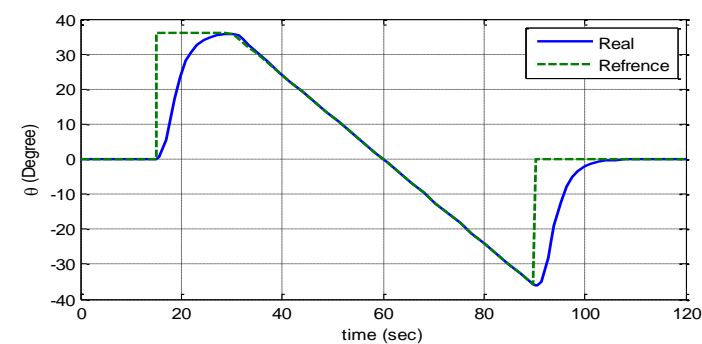

Figure 8. Extended LQR controller response

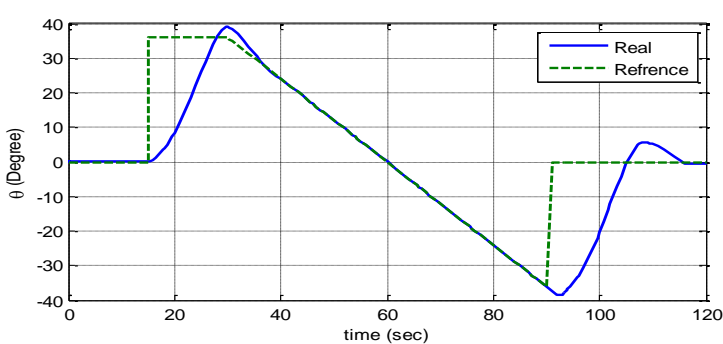

Figure 9. Fuzzy controller response

\section{Conclusion}

In this paper, different control algorithms has been evaluated for attitude tracking in push broom imaginary with forward motion compensator method. Reference signal for the control scenario was produced. Simulation results show that the Quaternion feedback PID controller and extended LQR have small time delay. An extended LQR controller for tacking has been designed with a new cost function with regard to reference state, that Compared to the other controllers, has a more appropriate response in the stabifization and target tracking are maneuverability. Designed Fuzzy controller has low steady state error in tracking the reference signal and has lowest torque control.

\section{References}

[1] J. R. Wertz and W. J. Larson, "Space Mission Analysis and Design", Third Edition, Microcosm Press, Hawthorne, CA, (1999).

[2] T. A. Sands, "Fine Pointing of Militaty Spacecraft", Dissertation, Naval Postgraduate School, Monterey, California, (2007) March.

[3] J. Oh, "Novel Approach to EpipolarResampling of HRSI and Satellite Stereo Imagery-based Geo-referencing of Aerial Images", The Ohio State University, Dissertation for the Degree Doctoral, (2011).

[4] D. Adams, D. Bruno, P. Shah and B. Keller "Precision Control, Knowledge and Orbit Determination on a Small Spacecraft Bus the OrbView-4 Attitude Control System", 12th AIAA Conference On Small Satellite, (2010).

[5] B. J. Kim and H. Lee, "Three Axis Reaction Wheel Attitude Control System For Kitsat-3 Microsatellite", Satellite Technology Research Center.

[6] M. J. Sidi, "Spacecraft Dynamics and Control, A Practical Engineering Approach", Cambridge University Press, (1997).

[7] P. Tisa and P. Vergez, "Performance Analysis of Control Algorithms for FalconSat-3", 16th AAS/AIAA Space Flight Mechanics Conference

[8] C. -H. Sheng and L. Shu, "Application of Fuzzy Controllers for Spacecraft Attitude Control", IEEE Transaction on Aerospace and Electronic Systems, vol. 45, no. 2, (2009) April.

[9] R. Sandau, "Satellite Earth Observation and Surveillance Payloads", German Aerospace Center, Rutherford str. 2, 12489 Berlin, Germany, RTO-EN-SCI-209.

[10] D. Poli, "Modelling of Spaceborne Linear Array Sensors", A dissertation submitted to the Swiss Federal Institute of Technology Zurich for the degree of Doctor of Technical Sciences, Zurich, (2005).

[11] "Pushbroøm/Whiskbroom Sensor Model", National Center for Geospatial Intelligence Standards, (2009) July 21.

[12] R. Gupta and R. Hartley, "Camera Estimation for Orbiting Pushbrooms", General Electric Corporate R\&D, River Rd_Schenectady_ NY-USA.

[13] L.Visagie, "A Bore-sight Motion Detection Algorithm for Satellite Attitude Control”, Stellenbosch University, (2007) December.

[14] S. Ge and H. Cheng, "A Comparative Design of Satellite Attitude Control System with Reaction Wheel", First NASA/ESA Conference on Adaptive Hardware and Systems, (2006) IEEE.

[15] D. Adams, D. Bruno, P. Shah and B. Keller "Precision Control, Knowledge and Orbit Determination on a Small Spacecraft Bus the OrbView-4 Attitude Control System”, 12th AIAA Conference On Small Satellite. 
[16] Kaplan, "LEO Satellites: Attitude Determination and Control Components; Some Linear Attitude Control Techniques", In Partial Fulfillment of the Requirements for the Degree of Master of Science in Electrical and Electronics Engineering, (2006) April.

[17] L. X. Wang, “A Course in Fuzzy Systems and Control”, Prentice-Hall international Inc., (1997).

\section{Authors}

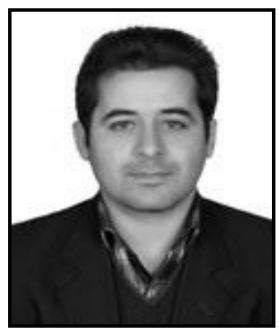

\section{Abbas Abdollahi Khoshmardan}

He was born in 1985 in Iran. He finished BS in Electrical Engineering/Control from Ferdowsi University of Mashhad (2007). He completed his post graduate in the Guidance and Control Systems (2011). He Interested in Aerospace Robotics and Intelligent Systems. He works on Satellite ADCS and Formation Flight Control now.

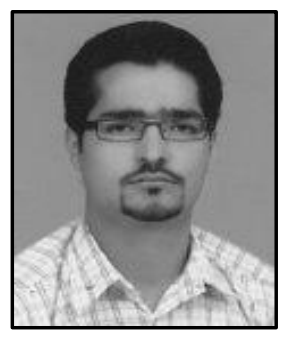

\section{Mohamad Reza Dastranj}

He finished him B.S from Islamic AZad University Sabzevar branch, Iran in 2008. He was graduated in Control Engineering, Islamic Azad University Gonabad branch Ian in $2011^{\circ}$ GONABAD. also interested in the field of evolutionary algorithms was the fractional-order systems. As a matter of fact, there are many paper published in international journal that confirm his expert in the the his research area.

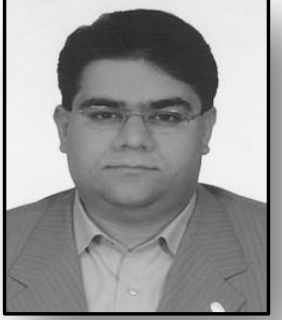

Aminreza Riahi

Aminreza Riahi rêceived the B.S. degree in control \& automation engineering in 2011 at the Islamic Azad University,Gonabad Branch.currently he is M.S. Student at the Islamic Azad University Gonbad Branch and a member of Young Researchers and Elite Club IAU in Iran. His research interests focus on evolutionary algorithms, Fractional-order systems and modern control engineering

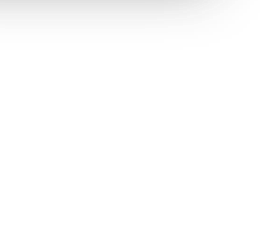

